\title{
Physics Reach of Electron-Capture Neutrino Beams*
}

\author{
J. Bernabeu ${ }^{\mathrm{a}}$, J. Burguet-Castell ${ }^{\mathrm{a}}$, C. Espinoza ${ }^{\mathrm{a}}$, M. Lindroos $^{\mathrm{b}}$ \\ ${ }^{\mathrm{a}}$ Universitat de Valencia and IFIC, E-46100 Burjasot, Spain. \\ ${ }^{\mathrm{b}}$ AB-department, CERN, Geneva, Switzerland.
}

\begin{abstract}
To complete the picture of neutrino oscillations two fundamental parameters need to be measured, $\theta_{13}$ and $\delta$. The next generation of long baseline neutrino oscillation experiments -superbeams, betabeams and neutrino factories- indeed take aim at measuring them. Here we explore the physics reach of a new candidate: an electron-capture neutrino beam. Emphasis is made on its feasibility thanks to the recent discovery of nuclei that decay fast through electron capture, and on the interplay with a betabeam (its closest relative).
\end{abstract}

\section{Introduction}

Electron Capture is a process in which an atomic electron is captured by a proton of the nucleus leading to a nuclear state of the same mass number $A$, replacing the proton by a neutron, and emitting an electron neutrino: $p e^{-} \rightarrow n \nu_{e}$.

It is a two body decay, and so the energy of the resulting neutrino is fixed. Thus, if we accelerate ions unstable by electron capture and let them decay in straight sections pointing to a far detector, we get a pure (that is, only one neutrino flavor) and monochromatic neutrino beam.

The rationale for doing this is much the same as for the $\beta$-beam, but with a nice extra feature which is to have a very peaked energy spectrum.

In reference [1] there is a more thorough discussion of the results we present here.

\section{Feasibility}

A possible implementation of the concept would involve the use of EURISOL to produce the unstable ions, the SPS to accelerate them, and a decay ring,

${ }^{*}$ Presented at the 7th International Workshop on Neutrino Factories and Superbeams (NuFact 05), Frascati, Italy, June 2005. The article will appear in the Conference Proceedings. 
Table 1: Decay properties of some rare-earth nuclei.

\begin{tabular}{|c|c|c|c|}
\hline Decay & $T_{1 / 2}$ & $E_{\nu}(\mathrm{keV})$ & $\mathrm{EC} / \beta^{+}(\%)$ \\
\hline${ }^{148} \mathrm{Dy} \rightarrow{ }^{148} \mathrm{~Tb}$ & $3.1 \mathrm{~m}$ & 2062 & $96 / 4$ \\
${ }^{150} \mathrm{Dy} \rightarrow{ }^{150} \mathrm{~Tb}$ & $7.2 \mathrm{~m}$ & 1397 & $99.9 / 0.1$ \\
${ }^{152} \mathrm{Tm} 2^{-} \rightarrow{ }^{152} \mathrm{Er}$ & $8.0 \mathrm{~s}$ & 4400 & $45 / 55$ \\
${ }^{150} \mathrm{Ho} 2^{-} \rightarrow{ }^{150} \mathrm{Dy}$ & $72 \mathrm{~s}$ & 3000 & $77 / 33$ \\
\hline
\end{tabular}

much like the one proposed for $\beta$-beams 3 . However, to allow electron capture to occur, we need to keep one electron bounded to the ion, and partly charged ions have a short vacuum life-time (even in a very good vacuum collisions with the few remaining atoms suffice to make them quickly lose its electron). So we need ions that decay fast enough.

Recent discovery of nuclei far from the stability line, having super enhanced spin-isospin transitions to a giant Gamow-Teller resonance kinetically accessible 2] turn out to be very good candidates. This discovery of nuclei that do decay fast enough through electron capture is the cornerstone that opens a window for a monochromatic neutrino beam experiment.

In Table 1 we show the properties of a few ion candidates.

\section{$3 \quad$ Experimental Setup}

For our simulations we have used a source of $10^{18}{ }^{150}$ Dy ions/year, during a total running time of 10 years.

In one case, we use 5 years at an ion acceleration of $\gamma=195$, the maximum achievable at CERN's SPS, and 5 years running at $\gamma=90$, as different as possible from the first one but still avoiding backgrounds in the detector. In a second case, we combine 5 years of electron capture at $\gamma=195$ with a 'standard' 5 years of $2.9 \times 10^{18}{ }^{6} \mathrm{He}$ ions/year $\beta^{-}$-beam.

Both scenarios have a 440 kton fiducial mass water Cerenkov detector located at a distance of $130 \mathrm{~km}$ (CERN-Frejus). Information from appearance and disappearance signals are combined.

\section{Electron Capture and $\beta^{ \pm}$-beam}

The main advantage of an electron capture beam over a $\beta$-beam, for a similar amount of ion decays, is that all the intensity is peaked at the energy(ies) of interest. In a $\beta$-beam the broad spectrum implies that many neutrinos will be produced at energies for which the dependence with $\delta$ is less pronounced, and/or the cross-section is too low.

On the other hand, it is not possible to produce an antineutrino beam with this technique. We can still combine runs at different energies to break the $\delta$ degeneracy, but the shape of the allowed regions after reconstruction at each energy is slightly similar, and so the intersection results in fits with sizable 

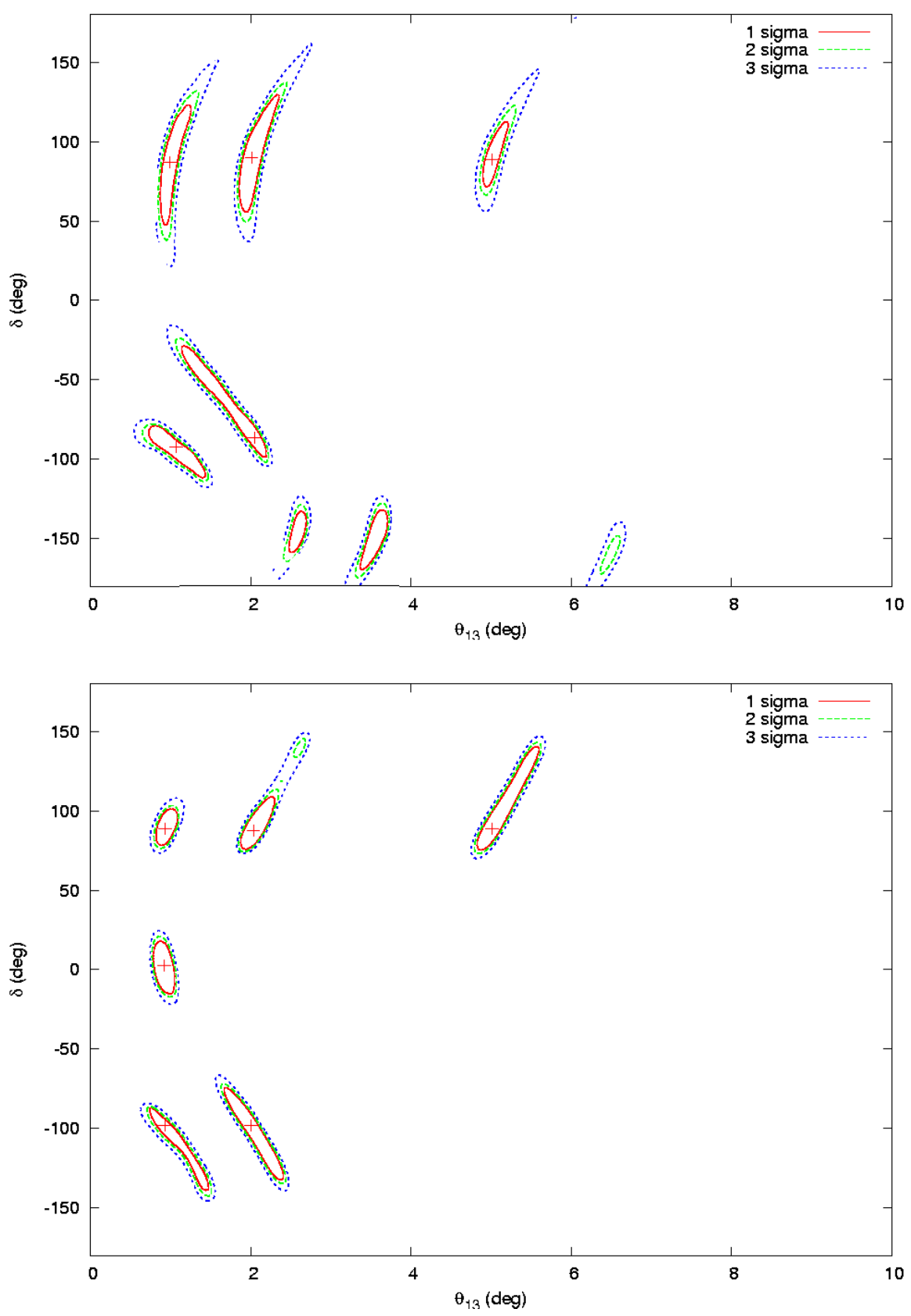

Figure 1: Combined fits of $\theta_{13}$ and $\delta$ for different central values of the parameters. The first (up) is a $5+5$ years e.c. at $\gamma=195,90$. It has worse resolution in $\delta$ and presents degeneracies. The second (down), $5+5$ years $\gamma=195$ and $\beta^{-}$-beam, show significantly better results. 
uncertainties in $\delta$. This affects to a (much) lesser amount to the determination of $\theta_{13}$. However, this also suggests that there must be a synergy between an electron-capture beam and a $\beta^{-}$-beam.

Figure (11) shows the results of the fits in both scenarios for certain chosen center values of the oscillation parameters. Figure (2) shows the sensitivity to a $\theta_{13} \neq 0$ achievable with the two electron capture scenario, which is quite impressive by itself.

Although not presented here, we have also done a preliminary study of the sensitivity to $\delta$. The scenario with two electron capture energies give poor results compared with standard proposed experiments, due to the similar oscillation dependence on $\delta$ even for quite different energies. On the other hand, the combination with a $\beta^{-}$-beam shows a very large sensitivity to the discovery of CP-violation, possibly beyond a Neutrino Factory, an effect mostly due to the allowed regions of each part intersecting more perpendicularly for low values of $\theta_{13}$, thus partially compensating for the simultaneous enlargement of these regions.

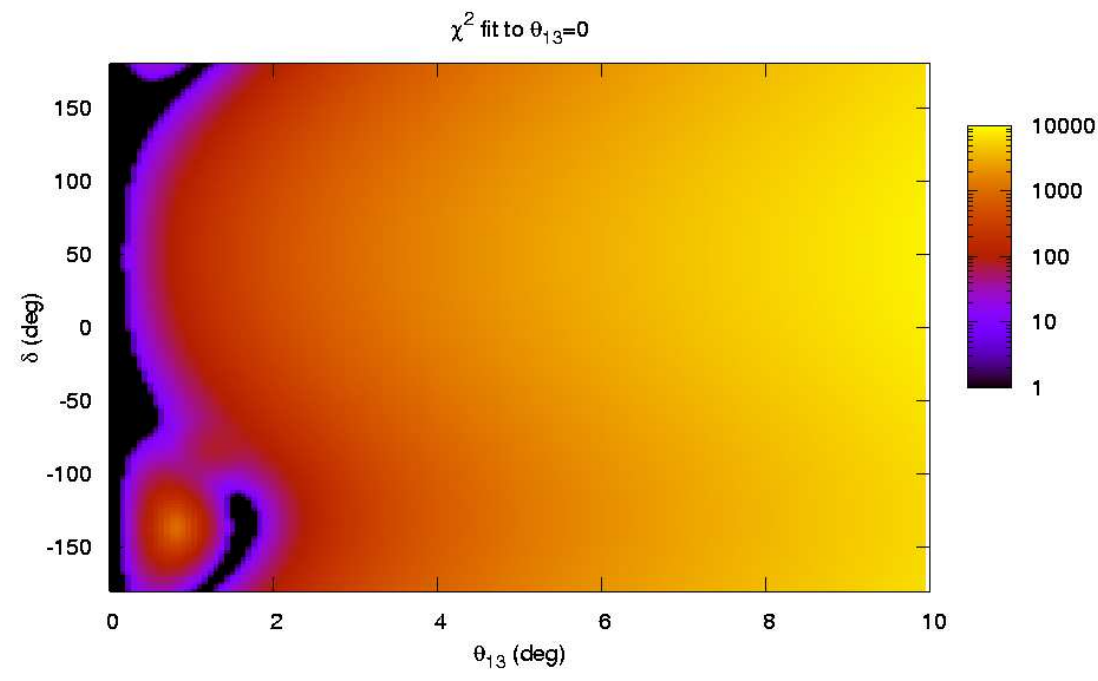

Figure 2: Sensitivity to $\theta_{13} \neq 0$ for $5+5$ years of e.c. at $\gamma=195,90$. Outside the black region a value of $\theta_{13}$ could be distinguished from 0 at $3 \sigma$ C.L. 


\section{Conclusion}

An electron capture neutrino beam is possible thanks to the recent discovery of heavy ions that decay fast through electron capture. Preliminary results on the sensitivity of such an experiment to $\theta_{13}$ and $\delta$ show its potential to compete with a Neutrino Factory, especially with the second scenario which merges neutrinos from electron capture with antineutrinos from a $\beta^{-}$-beam.

To reach a definite answer on the achievable flux a detailed study of production cross-sections, target and ion source designs, ion cooling and accumulation schemes, possible vacuum improvements and stacking schemes is required. Also, in order to the estimated sensitivity to stand, we need a careful study which includes the systematics due to the incomplete knowledge of other oscillation parameters and degeneracies due to the sign of $\Delta m_{23}^{2}$ and the quadrant of $\theta_{23}$.

Nevertheless, our results show the potential interest of the concept and hopefully encourage the further exploration we feel it deserves.

\section{References}

[1] J. Bernabeu, J. Burguet-Castell, C. Espinoza and M. Lindroos, arXiv:hep-ph/0505054

[2] A. Algora et al., Phys. Rev. C 70 (2004) 064301.

[3] B. Autin et al., J. Phys. G 29 (2003) 1785 arXiv:physics/0306106. 
This figure "ec_bb_fits.png" is available in "png" format from: http://arxiv.org/ps/hep-ph/0510278v1 
This figure "ec_ec_fits.png" is available in "png" format from: http://arxiv.org/ps/hep-ph/0510278v1 
This figure "sens_th13_chi2.png" is available in "png" format from: http://arxiv.org/ps/hep-ph/0510278v1 